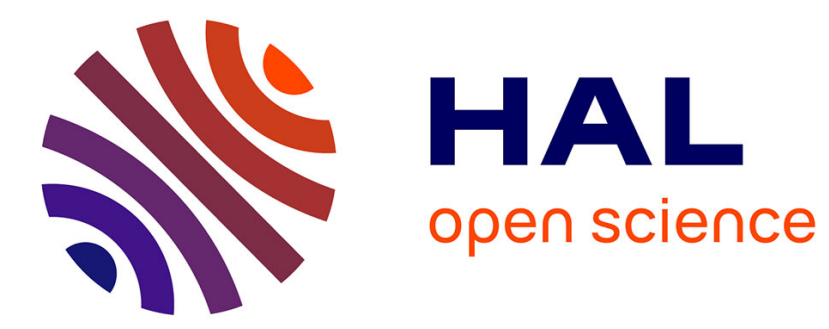

\title{
Contribution à l'étude du radical galvinoxyl par PDN
} J. Royaud

\section{To cite this version:}

J. Royaud. Contribution à l'étude du radical galvinoxyl par PDN. Revue de Physique Appliquée, 1971, 6 (2), pp.187-190. 10.1051/rphysap:0197100602018700 . jpa-00243521

\section{HAL Id: jpa-00243521 https://hal.science/jpa-00243521}

Submitted on 1 Jan 1971

HAL is a multi-disciplinary open access archive for the deposit and dissemination of scientific research documents, whether they are published or not. The documents may come from teaching and research institutions in France or abroad, or from public or private research centers.
L'archive ouverte pluridisciplinaire HAL, est destinée au dépôt et à la diffusion de documents scientifiques de niveau recherche, publiés ou non, émanant des établissements d'enseignement et de recherche français ou étrangers, des laboratoires publics ou privés. 


\title{
CONTRIBUTION A L'ÉTUDE DU RADICAL GALVINOXYL PAR PDN
}

\section{J. ROYAUD}

\author{
Laboratoire d'Optique Ultra-hertzienne de la Faculté des.Sciences de Bordeaux
}

\begin{abstract}
Résumé. - Le radical galvinoxyl est étudié à différentes concentrations en solution dans le diméthoxyéthane. La saturation électronique est réalisée à $10050 \mathrm{MHz}$ et la résonance nucléaire détectée à $15253 \mathrm{kHz}$. L'extrapolation des mesures, à puissance et à concentration infinies, conduit à un facteur de polarisation de 160 , ce qui suppose un paramètre de couplage de l'ordre de 0,25 , montrant que les conditions de ( rétrécissement extrême ) ne sont pas atteintes.
\end{abstract}

Abstract. - Galvinoxyl radical is investigated with different concentrations in solution in dimethoxyethane. Electronic saturation is realized at $10050 \mathrm{MHz}$ and the proton resonance is detected at $15253 \mathrm{kHz}$. The extrapolation of measurements at infinite concentration and power leads to a signal enhancement factor of 160 what implies a coupling parameter of about 0.25 . This shows that (cextreme narrowing) conditions are not achieved.

I. Spectre RPE du radical galvinoxyl. - Le radical galvinoxyl, ou "Coppinger's radical» a pour formule :<smiles>[2H]c1c(C(C)(C)C)cc(CC2C=C(C(C)(C)C)C(=O)C(C(C)(C)C)=C2)cc1C(C)(C)C</smiles>

GALVINOXYL

FIG. 1.

Son spectre RPE présente, en solution diluée, exempte d'oxygène, 9 raies de structure hyperfine qui s'expliquent par une séparation en deux niveaux due au couplage du spin électronique avec le spin nucléaire du proton de l'hydrogène central, chacun de ces niveaux étant ensuite décomposé, par couplage avec les 4 hydrogènes équivalents des cycles [1], en $2 n I+1=5$ sous-niveaux, dont les intensités relatives sont données par les coefficients du binôme $(1+x)^{4}$, soient : $1-4-6-4-1$. Les écarts hyperfins étant tels que deux raies se superposent, ce qui explique l'existence de 9 raies et les intensités obtenues (Fig. 2).

Int ensités

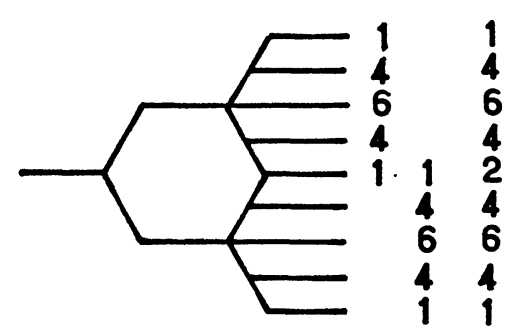

FIG. 2. - Décomposition hyperfine et intensités des raies du spectre RPE du galvinoxyl.
Il est intéressant de noter que j'ai obtenu un tel spectre (Fig. 3) avec une solution concentrée (M/2) de galvinoxyl dans le tétrahydrofuranne (THF), conservée en tube scellé, mais non dégazé, au bout de 9 jours.

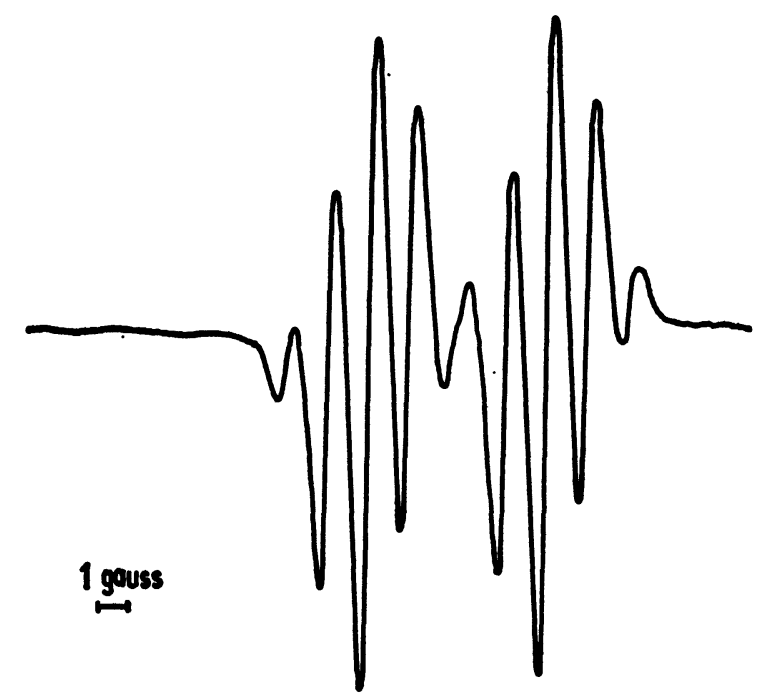

Fig. 3. - Enregistrement de la dérivée du spectre hyperfin du galvinoxyl dans le THF.

Ce résultat provient, sans doute, de la combinaison de l'oxygène dissous dans le solvant avec une partie du radical, ce qui a pour effet de diminuer la concentration et d'éliminer l'oxygène.

J'ai étudié l'évolution de la largeur de la raie (entre pics de la dérivée) des solutions de galvinoxyl dans le diméthoxyéthane (DME), en fonction de la concentration (Fig. 4).

Cette largeur diminue, quand la concentration augmente, et par suite de l'effet d'échange, tombe à 0,5 gauss pour $\mathrm{M} / 2$. 


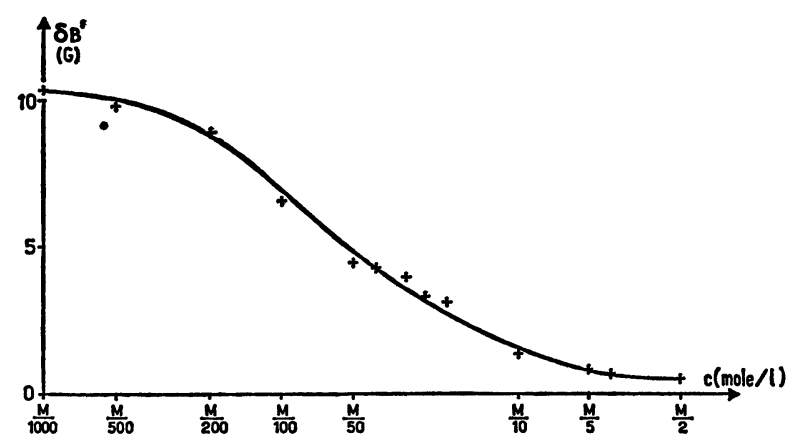

Fig. 4. - Largeur totale de l'enregistrement de la dérivée du spectre RPE de solutions de galvinoxyl dans le DME, en fontion de la concentration.

La figure 5 reproduit quelques enregistrements de spectres RPE, relatifs à des solutions concentrées de galvinoxyl dans le THF.
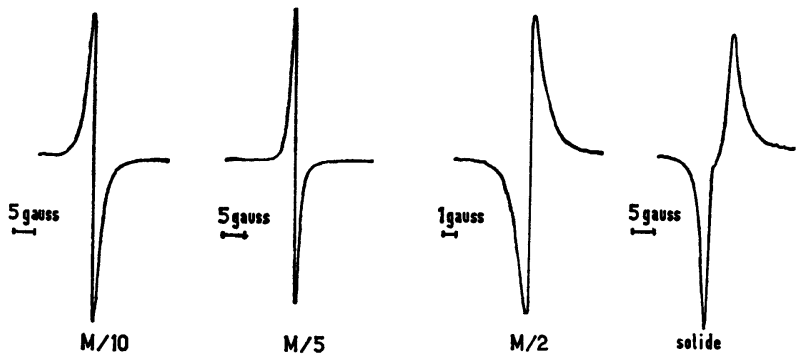

FIG. 5. - Enregistrements des dérivées des spectres RPE du galvinoxyl dans le THF, à différentes concentrations.

J'ai déterminé le facteur de décomposition spectroscopique $g=2,0045$, par une méthode de double enregistrement, décrite par ailleurs [2].

II. Saturation du radical galvinoxyl. - Les solutions concentrées de galvinoxyl se saturent facilement avec un klystron X 13, délivrant dans les conditions de l'expérience, une puissance de $320 \mathrm{~mW}$. On le voit sur l'exemple de la figure 6 , qui est relatif à une solution dont la largeur, à mi-hauteur, de la courbe d'absorption, était de $\delta B=2,4 \mathrm{G}$ et dont la forme lorentzienne permet de calculer un temps de relaxation transversal $\tau_{2}=2 / \gamma_{\mathrm{e}} \delta B=4,8 \times 10^{-8}$ s. Le mayimum de la courbe de la figure 6 correspondant à $P=50 \mathrm{~mW}$, soit à une amplitude du champ hyperfréquence dans la cavité rectangulaire $\mathrm{TE}_{012}$ utilisée, $B_{2}=0,1 \mathrm{G}$, ce qui permet de calculer un temps de relaxation spinmilieu $\tau_{1}=2,7 \times 10^{-6} \mathrm{~s}$, par application de la formule : $2 \tau_{1} \tau_{2} B_{2}^{2} \gamma_{\mathrm{e}}^{2}=1$.

Pour les solutions diluées, plus difficiles à saturer, j'ai utilisé un klystron Varian V 23, délivrant, dans les mêmes conditions, une puissance d'environ $3 \mathrm{~W}$. Sa fréquence étant asservie à celle de la cavité de mesure [3].

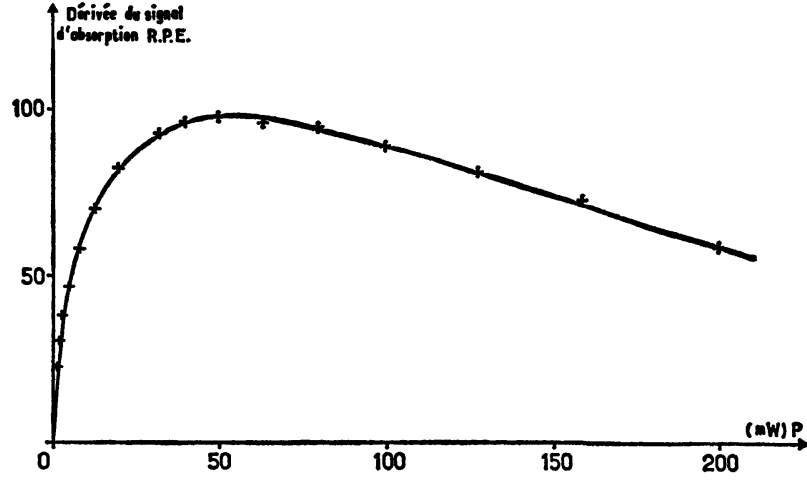

FIG. 6. - Maximum de la dérivée du signal d'absorption RPE d'une solution $\mathrm{M} / 5$ (âgée de 14 jours) de galvinoxyl dans le DME, en fonction de la puissance hyperfréquence.

III. Effet Overhauser inversé. - On observe la RMN à l'aide d'un oscillateur autodyne [4], fonctionnant à la fréquence de $v_{\mathrm{p}}=15253 \mathrm{kHz}$, celle du klystron étant réglée à $10050 \mathrm{MHz}$. On sature des solutions équilibrées, c'est-à-dire, dont l'oxygène a été, au moins partiellement éliminé ; la concentration réelle étant déterminée par comparaison avec un échantillon de DPPH.

Le facteur d'augmentation $a$ du signal RMN, augmente avec la concentration $c$ du radical. $a^{-1}$ est une fonction linéaire de $P^{-1}$ (Fig. 7). En effet :

$$
a=\frac{v-v_{0}}{v_{0}}=\xi f_{s} \frac{\gamma_{\mathrm{e}}}{\gamma_{\mathrm{p}}}
$$

où $: v$ est le signal RMN augmenté par saturation électronique,

$v_{0}$ est le signal RMN à l'équilibre thermodynamique,

$\xi$ est le paramètre de couplage,

$f$ est le facteur de fuite ;

$$
f=1-\frac{T_{1}^{\prime}}{T_{1}}
$$

où $T_{1}$ est le temps de relaxation spin-milieu“des protons du solvant pur ;

et $T_{1}^{\prime}$ le même temps en présence du radical; $\gamma_{\mathrm{e}}$ et $\gamma_{\mathrm{p}}$ sont les facteurs gyromagnétiques de l'électron et du proton ;

$s$ est le facteur de saturation :

$$
s=\frac{\gamma_{\mathrm{e}}^{2} B_{2}^{2} \tau_{1} \tau_{2}}{1+\tau_{2}^{2}\left(\omega-\omega_{\mathrm{e}}\right)^{2}+\gamma_{\mathrm{e}}^{2} B_{2}^{2} \tau_{1} \tau_{2}}
$$

d'où :

$$
\begin{gathered}
\frac{1}{a}=\frac{1}{\xi f \gamma_{\mathrm{e}} / \gamma_{\mathrm{p}}} \cdot \frac{1}{s}=\frac{1}{\xi f \gamma_{\mathrm{e}} / \gamma_{\mathrm{p}}} \cdot\left(1+\frac{1+\tau_{2}^{2}\left(\omega-\omega_{\mathrm{e}}\right)_{\mathrm{s}}^{2}}{\gamma_{\mathrm{e}}^{2} B_{2}^{2} \tau_{1} \tau_{2}}\right) \\
\frac{1}{a}=\frac{1}{a_{\infty}}\left(1+\frac{\alpha}{P}\right),
\end{gathered}
$$

$P$ étant proportionnelle à $B_{2}^{2}$. 
$a_{\infty}^{-1}$ étant obtenu par extrapolation à puissance infinie (intersection avec l'axe des ordonnées), ce qui revient à faire $s=1$. Quelques exemples sont montrés sur la figure 7.

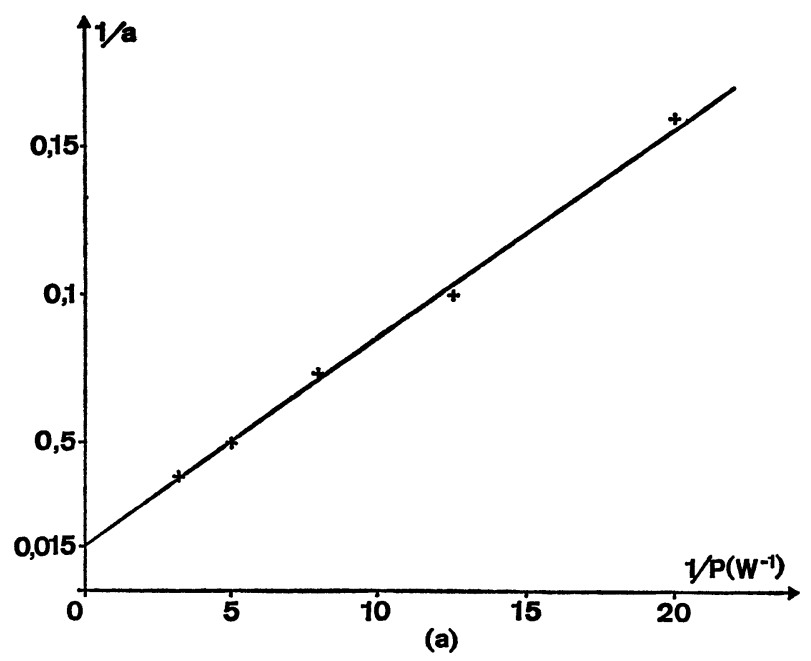

(a)

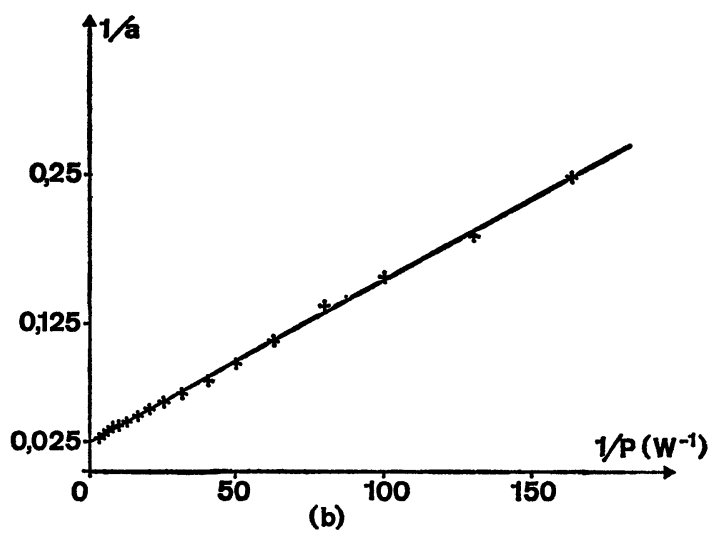

(b)

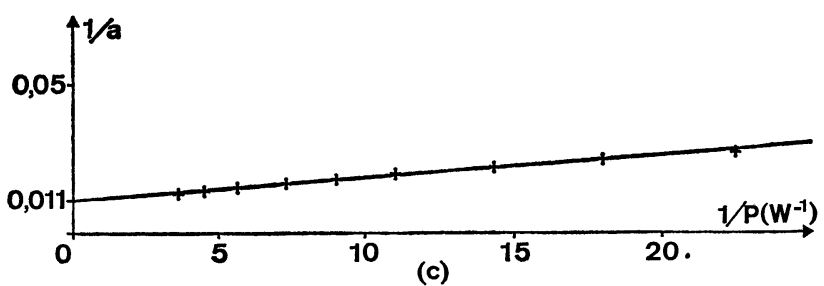

(c)

Fig. 7. $-a^{-1}$ en fonction du $P^{-1}$ pour différentes concentrations de galvinoxyl dans le DME : a) $\mathrm{M} / 10$; b) $\mathrm{M} / 20$; c) $\mathrm{M} / 5$.

On peut, de la même manière, s'affranchir du facteur de fuite, en traçant $a_{\infty}^{-1}$ en fonction de $c^{-1}$. On sait, en effet, que l'inverse du temps de relaxation des protons du solvant est une fonction linéaire de la concentration en impuretés paramagnétiques [6] et [7] :

$$
\frac{1}{T_{1}^{\prime}}=\frac{1}{T_{1}}+k c=\frac{1+k c T_{1}}{T_{1}}
$$

d'où

$$
\begin{gathered}
\frac{T_{1}^{\prime}}{T_{1}}=\frac{1}{1+k c T_{1}} \\
f=1-\frac{T_{1}^{\prime}}{T_{1}}=1-\frac{1}{1+k c T_{1}}=\frac{k c T_{1}}{1+k c T_{1}}
\end{gathered}
$$

et

$$
\frac{1}{a_{\infty}}=\frac{1}{\xi \gamma_{\mathrm{e}} / \gamma_{\mathrm{p}}}\left(1+\frac{1}{k c T_{1}}\right) \text {. }
$$

On voit, sur la figure 8 , que les valeurs de $a_{\infty}^{-1}$ obtenues à partir de la figure 7 , s'alignent sur une droite, qui coupe l'axe des ordonnées, pour la valeur $a$ (limite) $=-160$. Extrapoler, ainsi, à concentration infinie, revient à faire $f=1$.

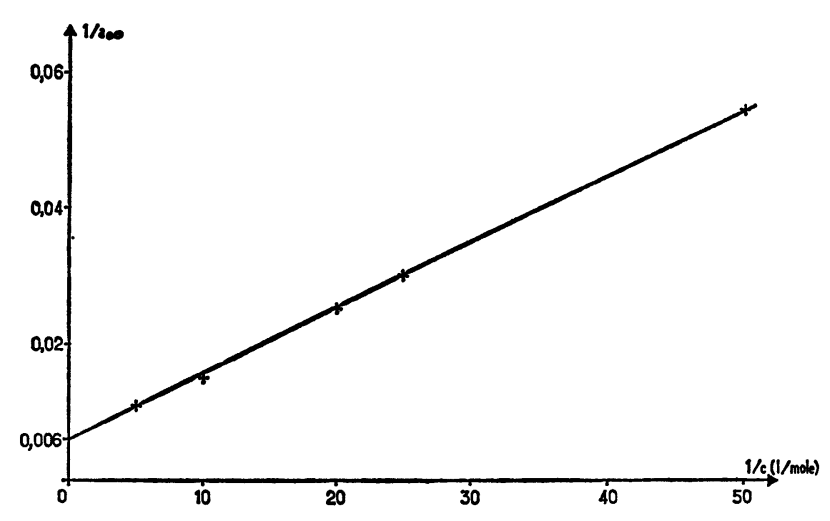

FIG. 8. $-a_{\infty}^{-1}$ en fonction de $c^{-1}$ pour le galvinoxyl dans le DME.

$\mathrm{La}$ formule $a=\xi f s \gamma_{\mathrm{e}} / \gamma_{\mathrm{p}}$, avec $s=f=1$ et $\gamma_{\mathrm{e}} / \gamma_{\mathrm{p}}=-660$ conduit à : $a$ (limite) $=-660 \xi$ $=-160$, d'où $\xi \# 0,25$. On sait que $\xi=0,5$ pour une interaction dipolaire, dans le cas du « rétrécissement extrême " $\left(\omega_{\mathrm{e}}^{2} \tau_{\mathrm{c}}^{2} \ll 1\right)$. Ce résultat implique que le temps de corrélation $\tau_{\mathrm{c}}$, pour le mouvement aléatoire produisant le couplage dynamique, est du même ordre de grandeur que la période des ondes hyperfréquences. Le paramètre de couplage $\xi$ décroit, en effet, en fonction de $\omega_{\mathrm{e}} \tau_{\mathrm{c}}$ [8]. Son expression est :

$$
\xi=\frac{6 J_{d}\left(\omega_{\mathrm{p}}+\omega_{\mathrm{e}}\right)-J_{d}\left(\omega_{\mathrm{p}}-\omega_{\mathrm{e}}\right)}{6 J_{d}\left(\omega_{\mathrm{p}}+\omega_{\mathrm{e}}\right)+J_{d}\left(\omega_{\mathrm{p}}-\omega_{\mathrm{e}}\right)+3 J_{d}\left(\omega_{\mathrm{p}}\right)}
$$

où les $J_{d}$ sont les fonctions de densité spectrale pour l'interaction dipolaire; elles peuvent s'exprimer en fonction d'un seul paramètre : le temps de corrélation $\tau_{\mathrm{c}} \mathrm{du}$ mouvement.

$$
J_{d}=\frac{\tau_{\mathrm{c}}}{1+\omega^{2} \tau_{\mathrm{c}}^{2}}
$$

dans notre cas $\omega_{\mathrm{e}} \gg \omega_{\mathrm{p}}$, d'où

$$
\xi=\frac{5}{10+3 \omega_{\mathrm{e}}^{2} \tau_{\mathrm{c}}^{2}},
$$

qui pour $\xi=0,25$ conduit à $\tau_{\mathrm{c}}=3 \times 10^{-11} \mathrm{~s}$ en bon accord avec la valeur trouvée par Kramer et Coll. [9]. 


\section{Bibliographie}

[1] WALKer and STRAW, ( Spectroscopy)), 1961, 1, 185

[2] Royaud (J.), “ Utilisation d'un spectromètre de double résonance pour la mesure du facteur de décomposition spectroscopique ). C. R. Acad. Sci. Paris, 1970, 271, 128.

[3] Royaud (J.), “ Asservissement de la fréquence d'un klystron à deux cavités, en vue d'études sur la double résonance ). Comptes rendus, 1968, 267B, 1018.

[4] Royaud (J.), ( Réalisation d'un oscillateur autodyne pour la résonance magnétique nucléaire ). ProcèsVerbaux, Soc. Sc. phys. nat. Bordeaux, 1963-64, 225.
[5] Hausser and Stehlik, " Dynamic Nuclear Polarization in Liquids ). Advances in Magnetic Resonance, 1968, 3, 85.

[6] Bloembergen, Purcell and Pound, Phys. Rev., 1948, 73, 679.

[7] Solomon (I.), « Relaxation Processes in a System of Two Spins ), Phys. Rev., 1955, 99, 559.

[8] Kruger, Muller-Warmuth and Van Steenwinkel, (( Molecular Motion in Liquids and Solutions ). Z. Naturforsch, 1966, 21a, 1224.

[9] Kramer, Muller-Warmuth and Schindler, J. Chem. Phys., 1965, 43, 31. 